\title{
Embedded Real Time Video Monitoring System using Arm
}

\author{
Kavitha Mamindla, Dr.V.Padmaja, CH.NagaDeepa \\ Department of Electronics and Communication Engineering, VNR Vignana Jyothi Institute of Technology, \\ Hyderabad, India.
}

\begin{abstract}
In this paper, Embedded Real-time video monitoring system based on ARM is designed, in which the embedded chip and the programming techniques are adopted. The central monitor which adopts S3C6410 chip as controller is the core of the whole system. First, USB camera video data are collected by the embedded Linux system, processed, compressed and transferred by the processing chip. Then, video data are sent to the monitor client by wireless network. Tests show the presented wireless video surveillance system is reliable and stable. And it has a perfect application prospects with real-time monitor.
\end{abstract}

Keyword: - Video Capture, Video compression, video streaming, server, Embedded Linux

\section{INTRODUCTION}

The Video surveillance [1] systems play an increasingly important role to maintain social security. It has been widely used in many fields, such as finance, public security, banking, and home. Traditional video surveillance can generally achieve close distance monitoring, by using the PC as a monitor host, monitor host connected monitor camera with coaxial cable. With the development of IT technology, especially the rapid development in embedded CPU of the open Linux systems, video conferencing, remote monitoring, and mass data processing fields can apply the embedded technology. This embedded monitoring system to overcome the week points of the traditional video surveillance systems, such as complex structure, poor stability, and expensive cost. Meanwhile, it possess some advantages, for example, hardware and software can be cut, compact construct, portable, low power consumption, more suitable for long-distance transmission. In this system, we use the high-performance S3C6410 chip as the microprocessor. Video data is captured from a USB camera, compressed into MPEG format, transferred the 3G network under the control of the ARM11 chip; then, the monitor client will receive the compressed data frame to restructure, and recompose video images .Wireless video monitor system provide a practical solution for remote wireless monitoring with low cost.

\section{HARDWARE SYSTEM DESIGN}

The hardware system includes processor, video-capture devices, and router to receive video information through Wi-Fi. In this paper SAMSUNG S3C6410 [2] ARM11 processor is chosen to complete the core control; Logitech CMOS camera is used as a video-capture device; and the user's phone or PC connected to the wireless Internet to receive video information to achieve real-time monitoring. As shown in Figure 1, block diagram of the hardware system design.

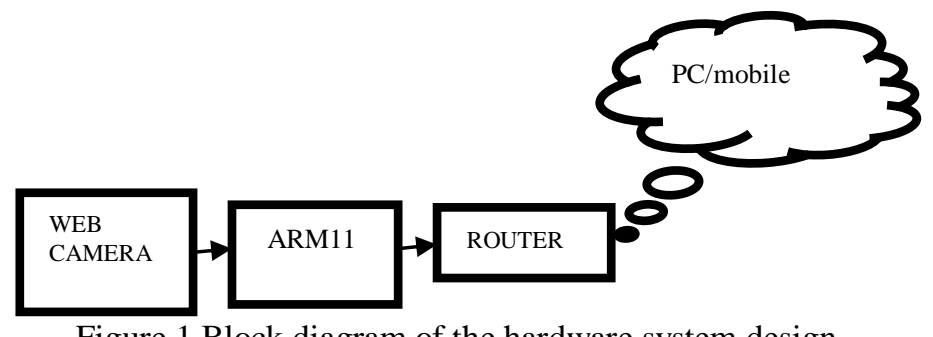

Figure 1.Block diagram of the hardware system design.

\subsection{SAMSUNG S3C6410 Processor}

The S3C6410 Board is a compact full-featured Embedded Single Board Computer (SBC) based upon Samsung ARM11 S3C6410, designed specifically for Mobile Internet Device, Notebook, handheld/3G mobile implementations. Its functional contents are similar to the latest generation of Pocket PC's and smart phones. This computer allows easy embedded application development through PC-Compatible tools and methods, while ensuring in-field reliability and ruggedness for end-user systems. S3C6410 runs without fans or heat sinks in the temperature range of $-20^{\circ} \mathrm{C}$ to $+70^{\circ} \mathrm{C}$. Other chip-level features include 4 UARTs, SPI, and I2C, a realtime clock with a separate power domain, and NAND Flash and DDR memory controllers. These features make the devices particularly suitable for automotive and industrial control applications as well as medical systems. In 
addition, the board supports Windows Embedded CE 6.0, Linux2.6, Android and Ubuntu. The Kit6410 Evaluation Board is developed based on Samsung ARM11 S3C6410X ARM1176JZF-S core 16/32-bit RISC microprocessor, which is designed to provide a cost-effective, low-power capabilities, high performance Application Processor solution for mobile phones ,notebook, industrial navigation system and general applications. To provide optimized $\mathrm{H} / \mathrm{W}$ performance for the $2.5 \mathrm{G} \& 3 \mathrm{G}$ communication services, the ARM chip S3C6410X adopts 64/32-bit internal bus architecture.

\subsection{Web Camera}

Logic tech camera with 1.3 million pixels is selected in the built system. Particularly in poor light, the speed of CMOS camera is slower, but its price very low, and CMOS has power consumption only when the circuit is connected, it is generally used low-end cameras, digital cameras and toys. There is a 20P plug with $2 \mathrm{~mm}$ pitch in ARM used as extension to connect the camera. This web camera continuously monitor the room and send the video.

\subsection{Router}

Router is a device that forwards the data packets between computer networks. Router is connected to S3C6410 ARM11 board through Ethernet cable. Using Wi-Fi we can stream the video through mobile or PC.

\section{SOFTWARE DESIGN}

The system selected Linux operating system [3][4] as software platform, the build environment using ARM-Gcc cross compiler debug mode, use embedded Linux 2.6 kernel. and there are mainly three function modules, that is, Video capture module, Video Compression module, and Video Streaming module. The flow chart system software shown in Figure 2.

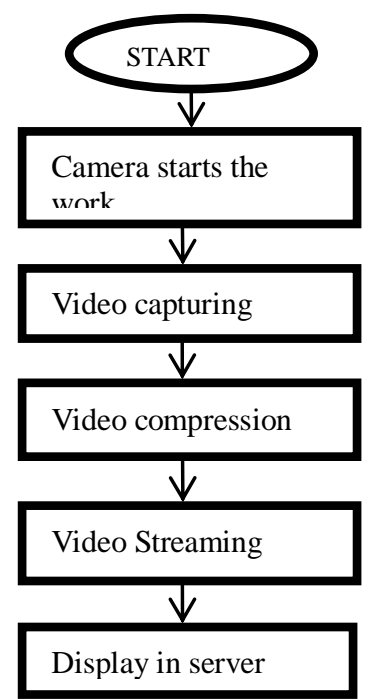

Figure 2: Flow chart of software system

\subsection{Video capture module}

Video Capture Based Video4Linux Video4Linux (referred to as "V4L") [1][5] is a Linux kernel on the video device driver, which is for video equipment, application programming interface functions to provide a system. The basic process of video capture shown in Figure 3. V4L USB camera using the programming on the need to use Linux system calls the next two, respectively, ioctl() and mmap().

Application to get the camera image data collected in two ways, namely read() (method of direct reading) and mmap() (memory mapping method). mmap() system call allows processes mapping the same file through memory sharing achieved, the advantages of high efficiency, because the process can directly read and write memory, copy any data without the need to speed up the I / O access, the system is Using this method.

\section{2 video compression}

The increasing demand to incorporate video data into telecommunications services, the corporate environment, the entertainment industry, and even at home has made digital video technology a necessity. A problem, however, is that still image and digital video data rates are very large, typically in the range of $150 \mathrm{Mbits} / \mathrm{sec}$. Data rates of this magnitude would consume a lot of the bandwidth, storage and computing resources in the typical personal computer. For this reason, Video Compression standards have been developed 
to eliminate picture redundancy, allowing video information to be transmitted and stored in a compact and efficient manner.

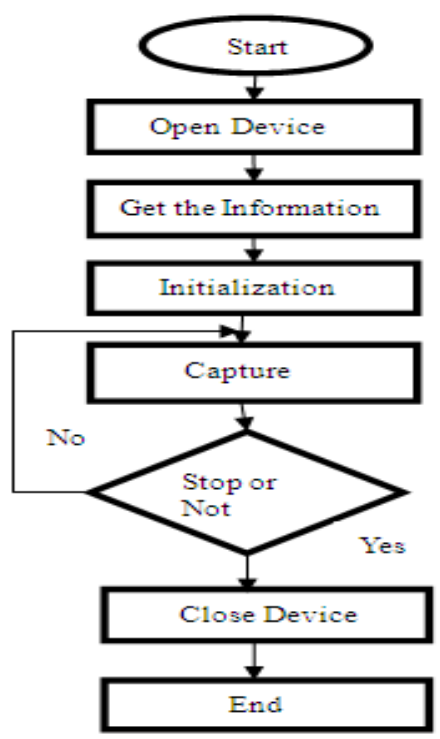

Figure 3.V4L Capture Flow

\subsubsection{Video Compression Standards}

Discrete Cosine Transform (DCT) based compression algorithms and international standards were developed to alleviate storage and bandwidth limitations imposed by digital still image and motion video applications.

Today there are three DCT-based standards that are widely used and accepted worldwide:

- JPEG (Joint Photographic Experts Group)

- H.261 (Video codec for audiovisual services)

- MPEG (Motion Picture Experts Group)

Each of these standards is well suited for particular applications: JPEG for still image compression, H.261 for video conferencing, and MPEG for high-quality, multimedia systems.

\subsubsection{MPEG-2 Compression Algorithm}

MPEG-2 provides for flexibility in the type of compression. Encoders can vary considerably depending upon the application, so details of the encoding scheme must be transmitted along with the data, to enable the decoder to reconstruct the signal. The fig.4.shows the flow of MPEG-2 Compression algorithm.[6][7].

In the figure 4 First a reduction of the resolution is done, which is followed by a motion compensation in order to reduce temporal redundancy. The next steps are the Discrete Cosine Transformation (DCT) and a quantization as it is used for the JPEG compression; this reduces the spatial redundancy (referring to human visual perception). The final step is an entropy coding using the Run Length Encoding and the Huffman coding algorithm.

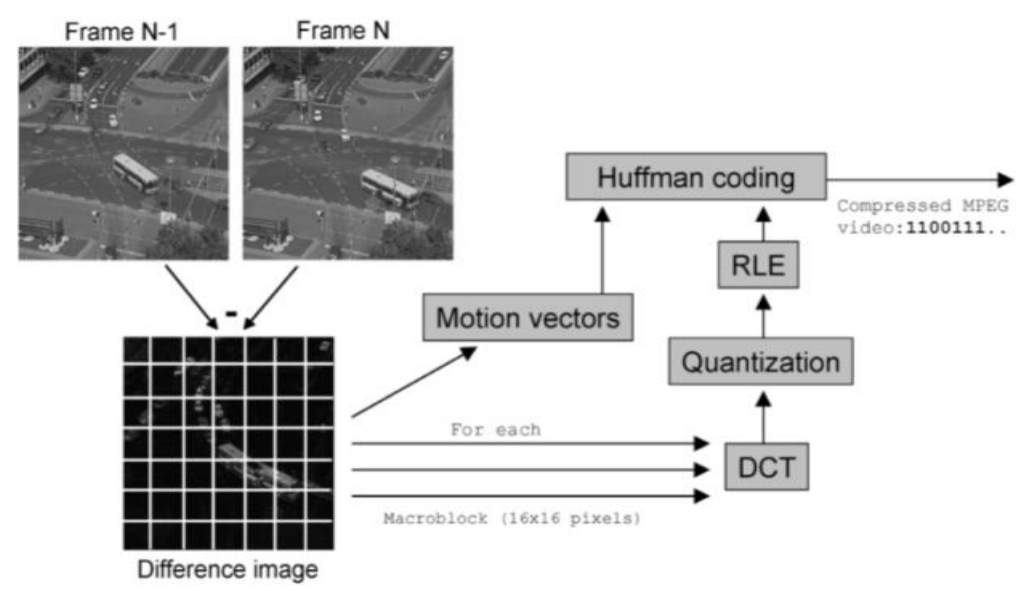

Figure 4.Flow of MPEG-2 Compression Algorithm. 


\section{Step 1: Reduction of the Resolution}

The human eye has a lower sensibility to colour information than to dark-bright contrasts. A conversion from RGB-colour-space into YUV colour components help to use this effect for compression. The chrominance components $\mathrm{U}$ and $\mathrm{V}$ can be reduced (sub sampling) to half of the pixels in horizontal direction (4:2:2), or a half of the pixels in both the horizontal and vertical (4:2:0).

\section{Step 2: Motion Estimation}

An MPEG-2 video can be understood as a sequence of frames. Because two successive frames of a video sequence often have small differences (except in scene changes), the MPEG-standard offers a way of reducing this temporal redundancy. It uses three types of frames:

I-frames (intra), P-frames (predicted) and B-frames (bidirectional). The I-frames are "key-frames", which have no reference to other frames and their compression is not that high. The P-frames can be predicted from an earlier I-frame or P-frame. P-frames cannot be reconstructed without their referencing frame, but they need less space than the I-frames, because only the differences are stored. The B-frames are a two directional version of the P-frame, referring to both directions (one forward frame and one backward frame). B-frames cannot be referenced by other P- or B- frames, because they are interpolated from forward and backward frames. P-frames and B-frames are called inter coded frames, whereas I-frames are known as intra coded frames.

\section{Step 3: Discrete Cosine Transform (DCT)}

DCT allows, similar to the Fast Fourier Transform (FFT), a representation of image data in terms of frequency components. So the frame-blocks ( $8 \times 8$ or 16x16 pixels) can be represented as frequency components.

\section{Step 4: Quantization}

During quantization, which is the primary source of data loss, the DCT terms are divided by a quantization matrix, which takes into account human visual perception. The human eyes are more reactive to low frequencies than to high ones. Higher frequencies end up with a zero entry after quantization and the domain was reduced significantly.

\section{Step 5: Entropy Coding}

The entropy coding takes two steps: Run Length Encoding (RLE ) [9] and Huffman coding [8]. These are well known lossless compression methods, which can compress data, depending on its redundancy, by an additional factor of 3 to 4 .

\subsection{Embedded Web Streaming Server}

Once RGB web camera is connected through master USB interface to arm board make minicom-s settings in the terminal window, during the settings we run the application related shell script in terminal which will execute application in board resulting video streaming on web browser using http protocol, entering a static IP address by user in any wireless device which is in local network can view the remote location.

Here the web browser is based on MJPG streamer for streaming captured video from camera placed in remote location. The MJPG streamer is cross-compiled and loaded in to the S3C6410 board to act as a web streaming server. The server periodically obtain videos from camera through the private network, such videos are transmitted from camera to the server. They are accumulated as MPEG video temporally into the internal buffer of the server.

The script file sh contains the following command :

cvlc vlc -vvv --color v412:///dev/video0:size=352x288:fps=25:adev=plughw:1,0 --sout "\#transcode $\{$ acodec $=$ mpga, $\mathrm{ab}=128$, samplerate $=48000$, channels $=1, \mathrm{vcodec}=\mathrm{mp} 2 \mathrm{v}, \mathrm{vb}=1000000\}:$ standard $\{$ access $=\mathrm{htt}$, mux $=\mathrm{t}$ $\mathrm{s}, \mathrm{dst}=192.168 .2 .5: 8080\}^{\prime \prime}$ on executing the script file the streaming process works.

Streaming server should support some default plug-in tools for streaming video like ffmpeg, vlc for displaying video consisting still of images..

VLC is the video LAN projects media player.VLC can also be used as a streaming server that duplicates the stream it reads and multicasts them through the network to other clients or serves them through http.

Gstreamer ffmpeg video plug-in supports a large number of audio and video compression formats through the use of FFMPEG library. Gstreamer is a streaming media framework which operates on media data. Application using this library can do anything from real-time sound processing to playing videos.

TCP/IP [5] application protocols included in the TCP/IP suite of protocols is an extensive list of applications designed to make use of the suites services. It is through these entities that resources can be made available data can be moved between hosts and remote user can communicate.

The processed stream data packets are uploaded into server using TCP/IP protocol and transmitted through wireless device so that user input the corresponding IP-address http://192.168.2.5/streamcam1.html and 
then the webpage is opened and the output video is streaming as shown in below Figure 5.

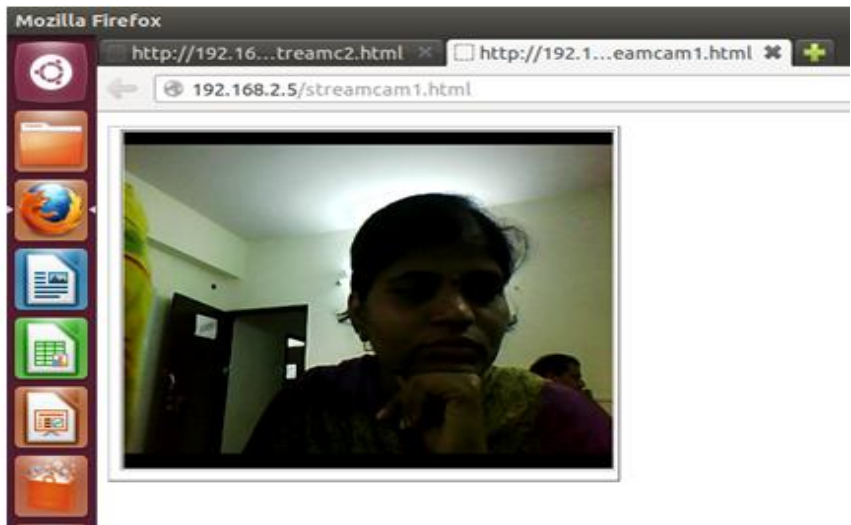

Figure 5.The output Video Streaming

\section{CONCLUSION}

In this paper, an embedded real-time monitoring system based on ARM was built, the embedded web streaming server is based on the ARM-Linux Operating System. It succeeds in network video monitoring. The system has low-cost, good openness and portability and is easy to maintain and upgrade.

This ARM based system, use CMOS image sensor to capture images, their colour and quality are acceptable, although imaging permeability and colour reproduction are not as CCD, it has advantages at low power consumption, price and integration. The video Compression module has good real-time processing performance and the saved video frames have high quality.

\section{REFERENCES}

[1]. Chen Rongfang, Wang Haibin, Hu Zhenhua, Zheng Pin. Remote Monitor System of Power Network Based on Embedded Internet Technology. Microcomputer Information. vol. 24, no. 7-2, pp. 78-80, 2008 S3C6410 Microcontroller User'sManualRevision1,http://www.samsungsemi.com/

[2]. Siyi.Zou, "Design and Application of Embedded Linux". Beijing: Tsinghua University Press, pp:9093,2002.

[3]. Zhang YuJie, Huai Weihua, Tian Ze. Design of Industrial Temperature Monitoring Equipment Based on Embedded Linux Operating System. Computer Engineering and Design, vol. 30, no. 17, pp. 3940-3942, 2009.

[4]. Video4Linux User's Manual, http://www.v4l.com.cn. [5] Craig Zacker. "TCP/ IP Administration”.China Water Power Press. Beijing, vol 8 ,pp:12- 18.1998.

[5]. Institute for Informatics- UniversityKarlsruhe. Http://www.irf.uka.de/seminaret/redundant/vortrag15/(3. Feb. 2006)

[6]. MANNING, C. The digital video site. http://www.newmediarepublic.com/video/compression/adv08.html (3. Feb. 2006)

[7]. HUFFMAN, D. A. (1951). A method for the construction of minimum redundancy codes. In the Proceedings of the Institute of Radio Engineers 40, pp. 1098-1101.

[8]. CAPON, J. (1959). A probabilistie model for run-length coding of pictures. IRE Trans. On Information Theory, IT-5, (4), pp. 157-163.

[9]. Yoshiro Imai, Yukio Hori : A Mobile Phone-Enhanced Remote Surveillance System with Electric Power Appliance Control and Network Camera Homing, IEEE Computer(Third International Conference on Autonomic and Autonomous Systems ICAS 2007). 